\title{
A Numerical Study of Rotational and Transverse Galloping Rectangular Bodies
}

\author{
I. Robertson ${ }^{\text {a }}$ L. Li ${ }^{\text {a,1 }}$ S. J. Sherwin ${ }^{\text {a,* }}$ P. W. Bearman ${ }^{\text {a }}$

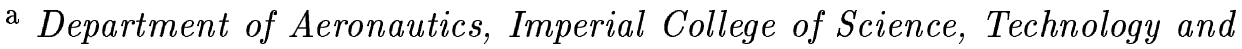 \\ Medicine, Prince Consort Road, London SW7 $2 B Y$, U.K.
}

\begin{abstract}
In this paper we investigate rotational and translational galloping instabilities due to fluid/structure interaction using a previously developed algorithm. This numerical technique utilises a two-dimensional spectral $/ h p$ element method and a frame of reference transformation to ensure efficient computations. Both transverse and rotational motion of rectangular sections of varying aspect ratio are simulated for a Reynolds number of 250 and at reduced velocities which promote a galloping response. Qualitative comparisons with quasi-steady theory and experimental data are found to be favourable.
\end{abstract}

\section{Introduction}

The study of flow-induced vibration of a structure is important in many fields including aeronautical, offshore, civil and mechanical engineering. The occurrence of flow-induced vibration is due to many different mechanisms, which are classified in terms of their predominant effect (Naudascher \& Rockwell, 1994; Deniz, 1997). Two well-known phenomena in the problems of fluid/structure interaction are vortexinduced vibration (VIV) and galloping. VIV is associated with synchronisation, or lock-in of the structural oscillation frequency with the vortex shedding frequency, while galloping is driven by a time-averaged fluid force which develops in phase with the structural velocity and has a frequency many times lower than that of vortex shedding. Lock-in occurs at reduced velocities where the vortex shedding frequency is comparable to the natural frequency of the structure, whereas galloping is prevalent at higher reduced velocities where the frequency of oscillation is lower than the vortex shedding frequency.

\footnotetext{
* Corresponding author.

1 Current address: Department of Mathematics, University College London, Gower Street, London WC1E 6BT, UK.
} 
Both mechanisms can lead to significant oscillation amplitudes, which can potentially result in catastrophic failure of a structure. A classic example of rotational galloping is the notorious collapse of the Tacoma Narrows Suspension Bridge, which was essentially due to a torsional instability (Steinman \& Watson, 1957; Scanlan, 1979; Scanlan \& Tomko, 1971). Transverse galloping oscillations can also be witnessed in winter when the build up of ice on long powerlines leads to the cables undergoing large amplitude, vertical oscillations.

Extensive work has been undertaken towards the investigation of the lock-in phenomena, which has been predicted by numerous numerical codes (Nomura, 1993; Wei et al., 1995; Anagnostopoulos, 1994; Schulz \& Kallinderis, 1998; Piperno, 1998; Li et al., 2002). Conversely, galloping instability has been the subject of very little reported research using numerical simulation.

The use of quasi-steady theory to predict whether a structure has the potential to gallop has been widely used for many years. It was developed by den Hartog (1956) and has generally been implemented to predict the critical reduced velocity for the onset of translational galloping instabilities. This has been verified experimentally by a number of investigators (Parkinson, 1971; Blevins, 1990; Nakamura \& Tomonari, 1977; Nakamura \& Nakashima, 1986; Nakamura \& Mizota, 1975). In this paper quasi-steady theory is used to determine the conditions under which rectangular structures of varying cross sectional area will undergo translational and rotational galloping and these predictions are compared with computationally derived results.

In Section 2 of the paper the solution method is outlined. Section 3 summarises the aerodynamic and structural equations and governing parameters. Section 4 contains an outline of the quasi-steady formulation of the galloping instability and comparison with numerical results. The conclusions constitute the final section.

\section{Solution Methods}

For the problem of an elastically mounted rigid body in a low speed flow, the governing equations are the incompressible Navier-Stokes equations with moving boundary conditions,

$$
\begin{gathered}
\nabla \cdot \mathbf{v}=0 \\
\frac{\partial \mathbf{v}}{\partial t}+(\mathbf{v} \cdot \nabla) \mathbf{v}=-\frac{1}{\rho} \nabla p+\nu \nabla^{2} \mathbf{v}, \quad \text { in } D(t),
\end{gathered}
$$

where $\mathbf{v}=\mathbf{v}_{b}(t) \quad$ on $\Gamma(t)=\partial D(t)$. Here $\mathbf{v}$ is the fluid velocity, $\rho$ is the density of the fluid, $p$ is pressure, $D(t)$ represents the moving fluid computational domain and $\Gamma(t)$ denotes the interface between the flow and the body and moves with an unknown velocity $\mathbf{v}_{b}$. 
For a single rigid body, its planar motion in two dimensions can be described in terms of three displacement components defined at the centre of gravity (Nomura $\&$ Hughes, 1992), $\mathbf{X}=(\zeta, \eta, \theta)^{T}$, where $\zeta$ and $\eta$ are the translational displacement components in the $x$ and $y$ direction, respectively, and $\theta$ denotes the rotational displacement component. We may write the governing equation for this linear, planar motion as

$$
\mathbf{M} \ddot{\mathbf{X}}+\mathbf{D} \dot{\mathbf{X}}+\mathbf{K X}=\overline{\mathbf{F}}
$$

where $\mathbf{M}, \mathbf{D}$ and $\mathbf{K}$ are the mass, damping and stiffness matrices, respectively, $\overline{\mathbf{F}}$ is a vector whose components are external forces and moment and a dot above a variable denotes differentiation with respect to time. The mounting system for the body is assumed to have both stiffness and damping.

Instead of directly solving the coupled system of equations (2) and (3), an efficient strategy is to solve the Navier-Stokes equations and structural equation explicitly and thereby decouple their solutions at each time step. This solution technique is commonly known as a loosely coupled algorithm and has been observed to be stable for cases where the structural density is much larger than the fluid density. Therefore, at each time level, we initially solve the Navier-Stokes equations to obtain the aerodynamic force acting on the body, then use the forces as an input in the structural solver to predict the displacement of the body at the next time level. This displacement is utilised as the boundary condition on the structure in the Navier-Stokes solver.

The most general and widely used method to simulate moving boundary problems is the Arbitrary Lagrangian-Eulerian formulation (ALE) where the computational mesh at the far-field boundaries is stationary and the nodes on the moving boundary take the same velocity as the structure (Nomura \& Hughes, 1992). The configuration of the computational mesh therefore changes at each time-step resulting in the Navier-Stokes solver constantly being regenerated, leading to large distortions of the numerical grid and preventing the use of efficient direct solvers for static meshes.

In $\mathrm{Li}$ et al. (2002), a numerical method is formulated which enables efficient computations by evaluating the governing two dimensional aerodynamic equations (1) and (2) on a fixed mesh. These equations are calculated in a moving frame of reference which is fixed to the structure and moves rigidly with it. The relationship between this moving frame of reference and a stationary fixed frame of reference is established, which leads to additional forcing terms in the Navier-Stokes equation (2). However, the extra computational cost in evaluating these additional forcing terms is small compared to that saved by evaluating the equations on a static mesh. 


\section{Model Equations}

We will consider the non-dimensional form of the Navier-Stokes equations:

$$
\begin{gathered}
\nabla^{*} \cdot \mathbf{v}^{*}=0 \\
\frac{\partial \mathbf{v}^{*}}{\partial t^{*}}+\left(\mathbf{v}^{*} \cdot \nabla^{*}\right) \mathbf{v}^{*}=-\nabla^{*} p^{*}+\operatorname{Re}^{-1}\left(\nabla^{*}\right)^{2} \mathbf{v}^{*} .
\end{gathered}
$$

The variables in the equations above have been non-dimensionalised as follows:

$$
\begin{gathered}
t^{*}=\frac{t U}{D}, \quad x^{*}=\frac{x}{D}, \quad y^{*}=\frac{y}{D}, \\
u^{*}=\frac{u}{U}, \quad v^{*}=\frac{v}{U}, \quad p^{*}=\frac{p}{\rho U^{2}} \\
\operatorname{Re}=\frac{\rho U D}{\mu}=\frac{U D}{\nu},
\end{gathered}
$$

where $D$ is a characteristic length such as the section depth, and $U$ is the freestream velocity respectively. Correspondingly, the forces and moment are non-dimensionalised by

$$
F_{x}^{*}=\frac{F_{x}}{\rho D U^{2}}, \quad F_{y}^{*}=\frac{F_{y}}{\rho D U^{2}}, \quad M_{\theta}^{*}=\frac{M_{\theta}}{\rho D^{2} U^{2}} .
$$

We note that the usual lift, drag and pitching moment coefficients are given by

$$
C_{x}=2 F_{x}^{*}, \quad C_{y}=2 F_{y}^{*}, \quad C_{M}=2 M_{\theta}^{*}
$$

The governing structural equation for the one degree-of-freedom transverse or heaving mode is

$$
m \ddot{y}+c_{y} \dot{y}+k_{y} y=F_{y},
$$

where $m$ is the mass per unit length of the body, $c_{y}$ is the damping constant, $k_{y}$ is the stiffness constant, and $y$ denotes the transverse location of the centre of the body. If we use the same non-dimensional scales as applied to the Navier-Stokes equations, then we obtain

$$
\ddot{y}^{*}+2 \xi_{y}\left(\frac{2 \pi}{U_{y}}\right) \dot{y}^{*}+\left(\frac{2 \pi}{U_{y}}\right)^{2} y^{*}=\frac{F_{y}^{*}}{n_{y}}=\frac{C_{y}}{2 n_{y}},
$$

where

$$
U_{y}=\frac{U}{f_{y} D} \quad(\text { reduced velocity }), \quad n_{y}=\frac{m}{\rho D^{2}} \quad(\text { mass ratio })
$$

and

$$
f_{y}=\frac{1}{2 \pi} \sqrt{\frac{k_{y}}{m}}(\text { natural frequency }), \quad \xi_{y}=\frac{c_{y}}{2 \sqrt{k_{y} m}} \text { (damping ratio ). }
$$

For the one degree-of-freedom torsional mode, equation (3) becomes

$$
I_{\theta} \ddot{\theta}+c_{\theta} \dot{\theta}+k_{\theta} \theta=M_{\theta}
$$


where $I_{\theta}$ is the mass moment of inertia, $c_{\theta}$ is the torsional damping constant, $k_{\theta}$ is the torsional stiffness constant, and $\theta$ denotes the rotational angle of the body around the elastic centre. The right-hand side of equation (13) is the moment about the elastic centre. The non-dimensional form of equation (13) is

$$
\ddot{\theta}+2 \xi_{\theta}\left(\frac{2 \pi}{U_{\theta}}\right) \dot{\theta}+\left(\frac{2 \pi}{U_{\theta}}\right)^{2} \theta=\frac{M^{*}}{n_{\theta}}=\frac{C_{M}}{2 n_{\theta}}
$$

where

$$
U_{\theta}=\frac{U}{f_{\theta} D}(\text { reduced velocity }), \quad n_{\theta}=\frac{I_{\theta}}{\rho D^{4}}(\text { mass moment of inertia ratio })
$$

and

$$
\left.f_{\theta}=\frac{1}{2 \pi} \sqrt{\frac{k_{\theta}}{I_{\theta}}}(\text { natural frequency }), \quad \xi_{\theta}=\frac{c_{\theta}}{2 \sqrt{k_{\theta} I_{\theta}}} \text { (damping ratio }\right) .
$$

We note that $f_{\theta}$ is the structural natural frequency for rotational motion. For the remainder of this paper, the structural equations will be used in their nondimensionalised form.

In general, if we denote the vibrational amplitude of a particular model by $A$, then the dimensionless amplitude of flow-induced vibration $A / D$ can be regarded as a function of four dimensionless parameters: (a) Reynolds number Re, as defined in equation (6); (b) Reduced velocity $U_{\text {red }}=U /\left(f_{n} D\right)$, where $f_{n}$ is the natural rotational or transverse structural frequency; (c) Mass ratio or mass moment of inertia ratio denoted by $n$; (d) Damping factor $\xi$.

We may write this function as

$$
\frac{A}{D}=\Theta\left(\operatorname{Re}, U_{\text {red }}, n, \xi\right)
$$

The most important task in the study of fluid/structure interaction is to determine the relationship between the dimensionless amplitude and the governing dimensionless parameters.

If we consider a series of rectangular cross-section bodies with varying thickness ratios $\Lambda=L / D$, where $L$ is the length of the body and $D$ its thickness, then equation (17) will also include a geometric parameter and becomes

$$
\frac{A}{D}=\Theta\left(\Lambda, \operatorname{Re}, U_{\text {red }}, n, \xi\right)
$$




\section{Quasi-Steady Analytical Formulation}

The theory related to galloping is based on a quasi-steady assumption; i.e., the fluid force on the structure is assumed to be determined solely by the instantaneous relative velocity and the angle of attack of the body (Blevins, 1990). Strictly this requirement is only satisfied at high reduced velocity, $U_{\text {red }}>20$ (Bearman, 1984), where the vortex shedding frequency is significantly greater than the natural frequency of the body. However, large amplitude responses do occur for lower reduced velocities (Blevins, 1990), for cases where the combined mass and damping is low. This response is generally caused by vortex shedding or some combination of vortex shedding and galloping. In order to capture pure galloping instabilities, we confine our numerical experiments to higher reduced velocities $U_{\text {red }} \geq 20$ in order to avoid the effect of vortex-induced resonance. It has been found that the thickness ratio of rectangular sections plays an important role in the mechanism of instability (Washizu \& Ohya, 1978; Washizu et al., 1980; Blevins, 1990; Nakamura \& Tomonari, 1977). For example, in their free oscillation experiment, Nakamura \& Tomonari (1977) found that soft transverse galloping, the spontaneous build up of oscillations from rest, only occurs for sections longer than a critical thickness ratio of 0.67 . In this paper the response of a range of rectangular sections at zero incidence with thickness ratios from 1 to 5 is investigated. The effect of increasing damping factor and mass ratio will also be considered.

\subsection{Heaving/Transverse Mode}

The following quasi-steady analysis to evaluate the aerodynamic damping on a heaving or transversely moving structure has been previously performed by many authors (Blevins, 1990; den Hartog, 1956) and is included here for completeness and to stimulate interest in the subsequent numerical experiments. Figure 1 illustrates a

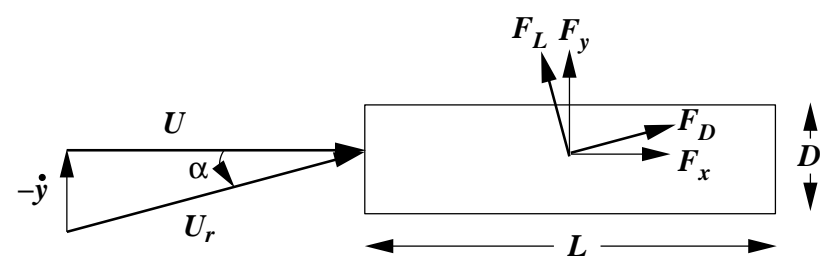

Fig. 1. Effective angle of incidence on a rectangular body

body oscillating in a vertical direction under the influence of forces generated by a horizontal steady fluid flow of velocity U. Transverse response of the body results in a velocity relative to the structure of $U_{r}$, at an effective angle of incidence $\alpha$, where

$$
U_{r}^{2}=U^{2}+\dot{y}^{2}
$$


and

$$
\tan \alpha=-\frac{\dot{y}}{U}
$$

Following aeronautical practise, the angle of incidence is positive for a clockwise rotation of the body in a flow travelling from left to right.

The force on the body per unit length is

$$
F_{y}=\frac{1}{2} \rho U^{2} D C_{y},
$$

where $C_{y}$ is the vertical or normal force coefficient which is related to the lift and drag coefficients, $C_{L}$ and $C_{D}$ respectively, by

$$
C_{y}=\frac{U_{r}^{2}}{U^{2}}\left(C_{L} \cos \alpha+C_{D} \sin \alpha\right)
$$

Due to the nature of the quasi-steady analysis the normal force, lift and drag coefficients used in equation (22) should be taken as time-averaged values, where the variation of these coefficients due to high frequency effects are ignored. Though it is not explicitly illustrated, subsequent appearances of these force coefficients should be read as time-averaged values. For small effective angles of attack, equation (20) can be approximated by

$$
\alpha=-\frac{\dot{y}}{U}
$$

and $C_{y}$ can be expanded about $\alpha=0$, resulting in the relationship,

$$
\left.C_{y} \cong C_{y}\right|_{\alpha=0}+\left.\frac{\partial C_{y}}{\partial \alpha}\right|_{\alpha=0} \alpha
$$

Assuming $\left.C_{y}\right|_{\alpha=0}=0$ and using equation (23) gives

$$
C_{y} \cong-\left.\frac{\partial C_{y}}{\partial \alpha}\right|_{\alpha=0} \frac{\dot{y}}{U}
$$

By utilising the representation of $C_{y}$ in equation (25), we can formulate an expression for the vertical motion of the body,

$$
\ddot{y}^{*}+2 \xi_{y}\left(\frac{2 \pi}{U_{y}}\right) \dot{y}^{*}+\left(\frac{2 \pi}{U_{y}}\right)^{2} y^{*}=-\left.\frac{1}{2 n_{y}} \frac{\partial C_{y}}{\partial \alpha}\right|_{\alpha=0} \dot{y}^{*}
$$

Considering equation (26) the net damping of the system, $\Psi_{y}$, can be shown to be

$$
\Psi_{y}=2 \xi_{y}\left(\frac{2 \pi}{U_{y}}\right)+\left.\frac{1}{2 n_{y}} \frac{\partial C_{y}}{\partial \alpha}\right|_{\alpha=0}
$$



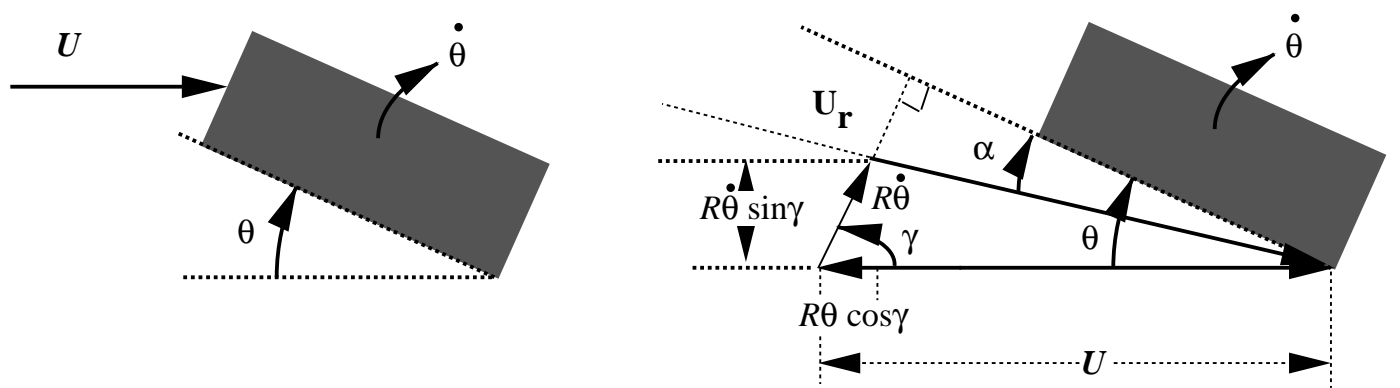

Fig. 2. Analytical model used in quasi-steady analysis of rotational galloping

where $\Psi_{y}<0$ will result in galloping oscillations, since the solution of equation (26) would be associated with exponentially growing oscillatory solutions. The structural damping, $\xi_{y}$, is always expected to be positive, therefore a necessary condition for instability is

$$
\left.\frac{\partial C_{y}}{\partial \alpha}\right|_{\alpha=0}<0
$$

\subsection{Torsional/Rotational Mode}

Similar to the analysis of the transverse mode, the net damping on a rotating structure, can be approximately evaluated using quasi-steady theory. Again this analytical investigation has been performed previously and the subsequent derivation mainly follows that of Blevins (1990). As we have seen, the relative angle of attack of a body vertically oscillating is proportional to its vertical velocity, here it will be shown that for a rotating body the relative angle of attack is proportional to its physical angle of attack, $\theta$, and its rotational velocity, $\dot{\theta}$.

The relative angle of attack, $\alpha$ and relative velocity, $U_{r}$, at a radius $R$ from the centre of rotation of a rotating body can be evaluated as

$$
\begin{gathered}
\alpha=\theta-\tan ^{-1}[R \dot{\theta} \sin \gamma /(U-R \dot{\theta} \cos \gamma)] \\
U_{r}^{2}=(R \dot{\theta} \sin \gamma)^{2}+(U-R \dot{\theta} \cos \gamma)^{2}
\end{gathered}
$$

where the variables are defined in Figure 2 and $\gamma=\pi / 2-\theta$. The effective angle of attack, $\alpha$, is obviously dependent on the physical angle of attack, $\theta$, though there is also a contribution due to the rotational velocity of the body.

The rotational motion at the leading edge of the body results in a decrease of the relative free-stream velocity in the horizontal direction. Therefore $(U-R \dot{\theta} \cos \gamma)$ is the velocity of the horizontal free stream when the rotational velocity of the body is considered. The vertical motion of the leading edge can be related to the quasisteady theory for purely heave or transverse motion where a non-dimensional positive 
transverse velocity was shown to be equivalent to a negative effective angle of attack. The leading edge of the body, for small oscillations about a mean displacement, can in a linear sense be said to be undergoing purely vertical motion. As with the transverse theory this vertical motion can be related to an effective angle of attack.

The length $R$ is a characteristic radius at which the relative angle of attack due to the rotational velocity of the body is considered. This technique has been used extensively for many different geometries (Blevins, 1990; Nakamura \& Mizota, 1975; Sisto, 1953) and in the case of rectangular sections, $R$ is taken to be the half-chord length, D/2 (Blevins, 1990; Nakamura \& Mizota, 1975). This choice of $R$ results in the instantaneous relative angle of attack, $\alpha$, being evaluated at the leading edge where the most significant forces for rotational motion are generated. For small angles and velocity equations (29) and (30) reduce to

$$
\begin{aligned}
\alpha & \approx \theta-\frac{R \dot{\theta}}{U}, \\
U_{r} & \approx U .
\end{aligned}
$$

Therefore the time-averaged value of $C_{M}$ can be expanded about $\alpha=0$, resulting in the relationship

$$
\begin{aligned}
C_{M} & \left.\cong \frac{\partial C_{M}}{\partial \alpha}\right|_{\alpha=0} \alpha \\
& \left.\cong \frac{\partial C_{M}}{\partial \alpha}\right|_{\alpha=0}\left(\theta-\frac{R \dot{\theta}}{U}\right)
\end{aligned}
$$

The same method adopted for the transverse galloping analysis is now applied to formulate a condition which will determine the potential of a body to undergo rotational galloping. An equation of motion governing the rotation of the body can be formulated by combining the structural equation (14) and the expansion of $C_{M}$ in equation (34) to arrive at

$$
\ddot{\theta}+2 \xi_{\theta}\left(\frac{2 \pi}{U_{\theta}}\right) \dot{\theta}+\left(\frac{2 \pi}{U_{\theta}}\right)^{2} \theta=\left.\frac{1}{2 n_{\theta}} \frac{d C_{M}}{d \alpha}\right|_{\alpha=0}\left(\theta-R^{*} \dot{\theta}\right)
$$

where $R^{*}=R / D$. Considering equation (35) the net damping of the system, $\Psi_{\theta}$, is

$$
\Psi_{\theta}=2 \xi_{\theta}\left(\frac{2 \pi}{U_{\theta}}\right)+\left.\frac{R^{*}}{2 n_{\theta}} \frac{d C_{M}}{d \alpha}\right|_{\alpha=0}
$$

The condition $\Psi_{\theta}<0$ will result in rotational galloping oscillations. As the structural damping for rotational motion, $\xi_{\theta}$, is always expected to be positive, a necessary 

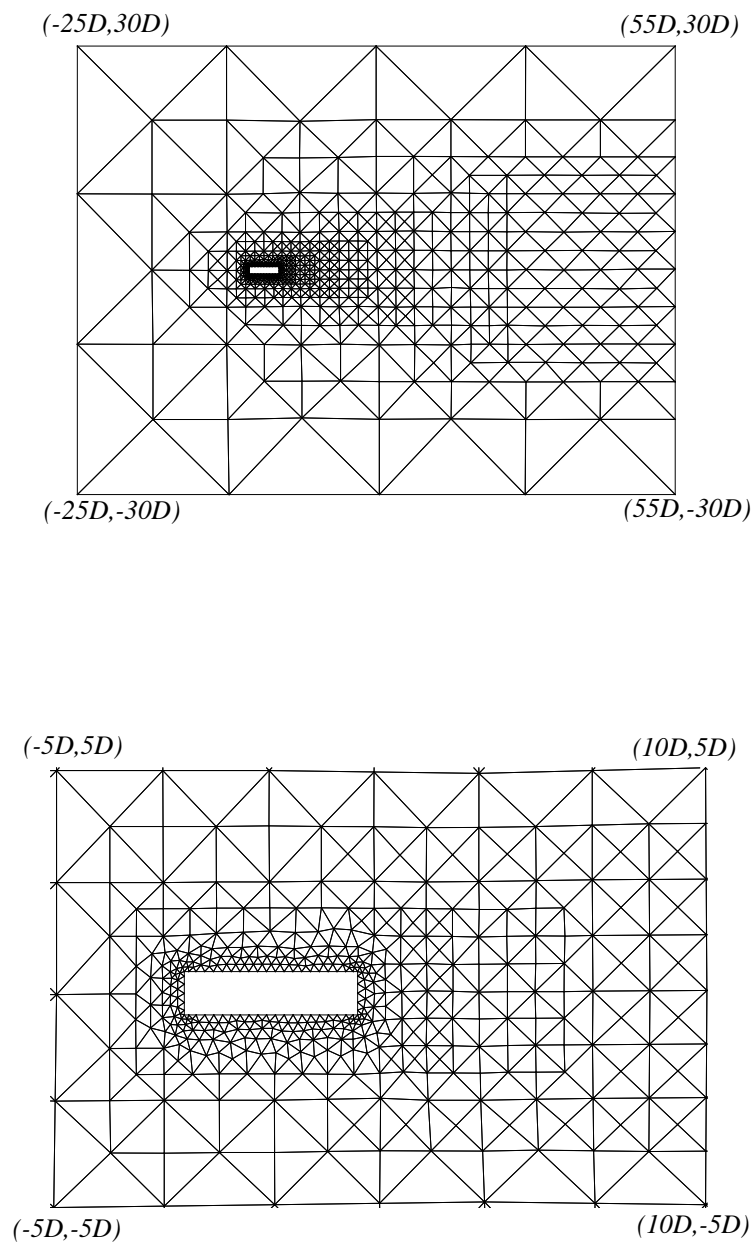

Fig. 3. Mesh configuration for numerical experiments condition for instability under this linearised analysis is

$$
\left.\frac{\partial C_{M}}{\partial \alpha}\right|_{\alpha=0}<0 .
$$

when $\alpha \ll 1$.

\section{$5 \quad$ Numerical Investigation of Transverse Galloping}

To enable a prediction of the instability of a series of rectangular sections with increasing thickness ratio $\Lambda$, a number of numerical experiments were conducted to evaluate the force coefficients $C_{y}$, for a range of angles of attack. The full computational mesh and an enlargement centred on the body for a rectangular structure with $\Lambda=4$ can be seen in Figure 3. The mesh consists of 1303 high order sub-domains and the computations were carried out using a polynomial expansion basis of order 
(a)

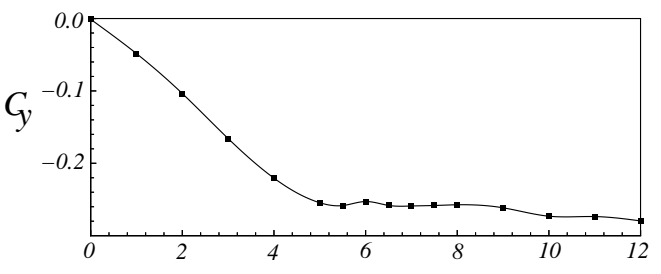

(c)

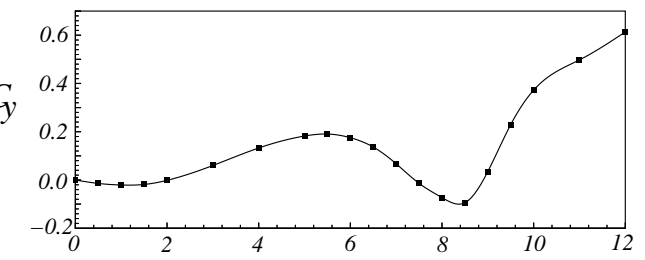

(d)

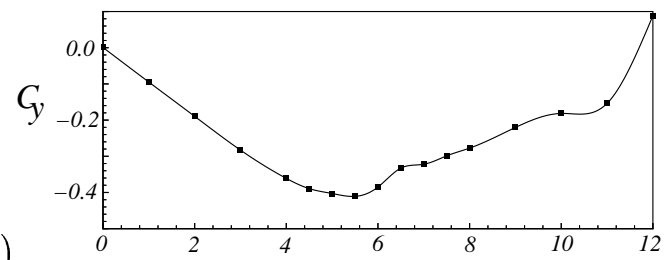

(b) (e)

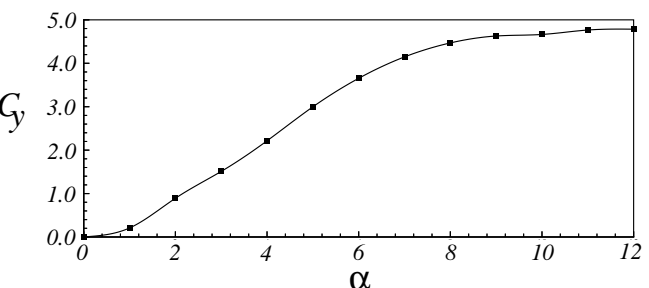

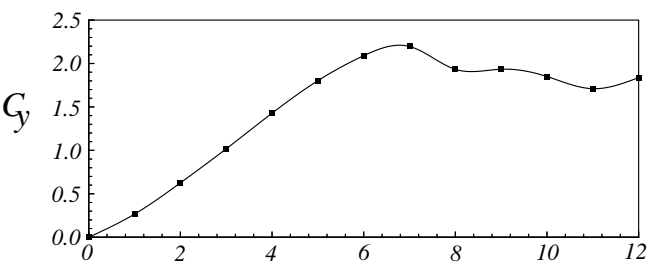

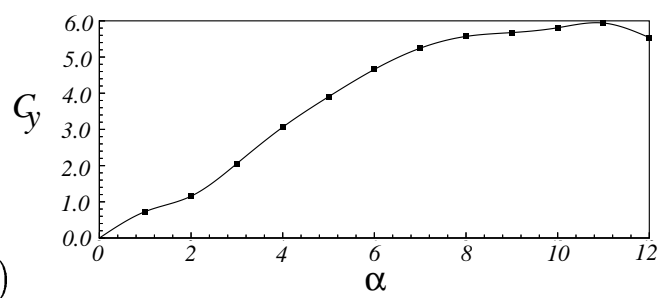

Fig. 4. Lift force coefficients against the angle of attack $\alpha$ (degrees) for different thickness ratios $(\Lambda)$ of rectangle cylinders. (a) $\Lambda=1$. (b) $\Lambda=1.5$. (c) $\Lambda=2$. (d) $\Lambda=3$. (e) $\Lambda=4$. (f) $\Lambda=5$.

5. Spatial and temporal convergence tests for an oscillating section can be found in Appendix A.

The results for thickness ratios ranging from 1 to 5 can be seen in Figure 4, where $C_{y}$ is represented as a function of $\alpha$. The values of $d C_{y} /\left.d \alpha\right|_{\alpha=0}$ are contained in Table 1.

According to the quasi-steady theory, if the variation of force coefficient with angle of attack has a negative slope around the origin, the corresponding structure is potentially unstable to transverse galloping. Therefore by studying the results in Figure 4 and Table 1, the structures with thickness ratios $\Lambda=1$ (square section), 1.5 and 2 may be susceptible to transverse galloping. To test this hypothesis these cases were simulated numerically by subjecting the sections to a fluid flow parallel to the $x$-axis and allowing them to oscillate with fluid and structural parameters set to: $\xi_{y}=0.0037, U_{y}=40, n=20$ and $\operatorname{Re}=250$.

The results are presented in Figure 5, where as expected the rectangular sections with thickness ratios $\Lambda=1$ and $\Lambda=1.5$ experience large amplitude oscillations (approximately $100 \%$ of the thickness of the cylinder). From the plots shown in Figures 4(a) and (b), we see that as the angle of attack increases, it eventually enters a stable range, from the point of view of the quasi-steady analysis. Therefore we expect an increase in effective angle of incidence to lead to a reduction in the magnitude of the applied aerodynamic force. Applying the quasi-steady analysis in 
Table 1

\begin{tabular}{|c||c|c|c|c|c|c|}
\hline$\Lambda$ & 1 & 1.5 & 2 & 3 & 4 & 5 \\
\hline$\left.\frac{d C_{y}}{d \alpha}\right|_{\alpha=0}$ & -2.69 & -5.44 & -1.60 & 14.4 & 7.39 & 44.8 \\
\hline
\end{tabular}

Variation of the lift coefficient against angle of attack in radians about a zero effective angle of attack for rectangular sections of increasing aspect ratio.

this manner suggests a mechanism as to why the large oscillation amplitudes due to the galloping instability can be sustained without growing unbounded.

For a thickness ratio of $\Lambda=2$, the amplitude of the oscillations is reduced (approximately $0.015 \%$ of the thickness of the cylinder), though low frequency oscillations are observed, indicating a galloping motion. Superimposed on this low frequency modulation is a high frequency motion due to vortex shedding. The low recorded amplitude is possibly due to the fact that the instability range and magnitude of $d C_{y} / d \alpha$ for this case is very small, see Figure $4(\mathrm{c})$ and Table 1.

For larger $\Lambda$, transverse galloping is suppressed, as predicted by the quasisteady theory. These numerical results are consistent with the experimental results of Washizu \& Ohya (1978), which show that no transverse galloping exists for $\Lambda>2.5$. At higher values of $\Lambda$ only vortex-induced oscillations are observed, which is illustrated in the response time history plotted in Figure 5(d). The agreement between the experimental results of Washizu et al. (1980) and the current numerical results may be surprising when considering the disparity in Reynolds number. The experiments were conducted at a Reynolds number of $\approx 1 \times 10^{5}$ while the numerical results were performed at $\operatorname{Re}=250$. However, we note that sharp edged bodies such as the rectangular sections have well-defined separation points which show no significant movement for changes in Reynolds number. The re-attachment of the separated shear layers is Reynolds number dependent, though it appears from the agreement between the experimental and numerical results that this difference has little effect on the stability or otherwise of the sections. Another significant difference between the experimental and numerical results is the restriction of the numerical experiments to two-dimensional flow. It has been shown that this restriction has some effect on the quantitative comparison of the amplitude of the unstable motions, but less effect on the point of instability (Robertson et al., 2002, 2001). At low oscillation amplitudes, that is those prior to the galloping instability, the vortical structures produced experimentally may have a finite correlation length implying a decorrelation of the forces and moments along the length of the bridge deck section. It has been shown for circular cylinders that when the oscillations increase in size the vortical structures become homogeneous along the length of the body (Kozakiewicz et al., 1992; Sumer et al., 1994). In summary the three-dimensionality of the physical experiments can have an effect on the quantitative oscillation amplitudes, without affecting the reduced velocity for the onset of galloping. 


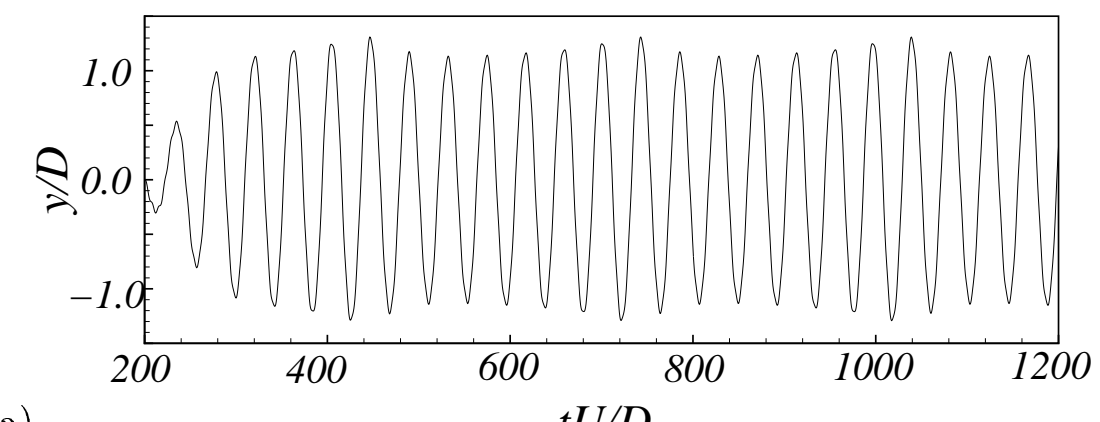

(a)

$$
t U / D
$$

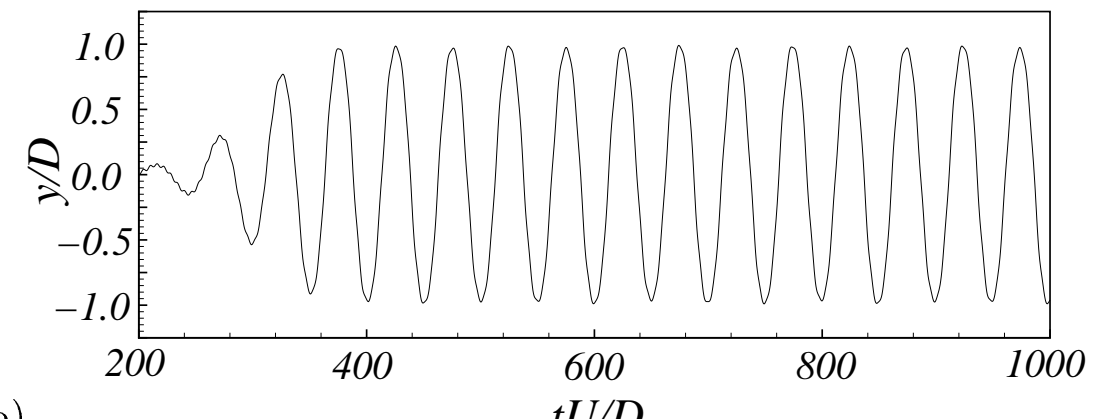

(b)

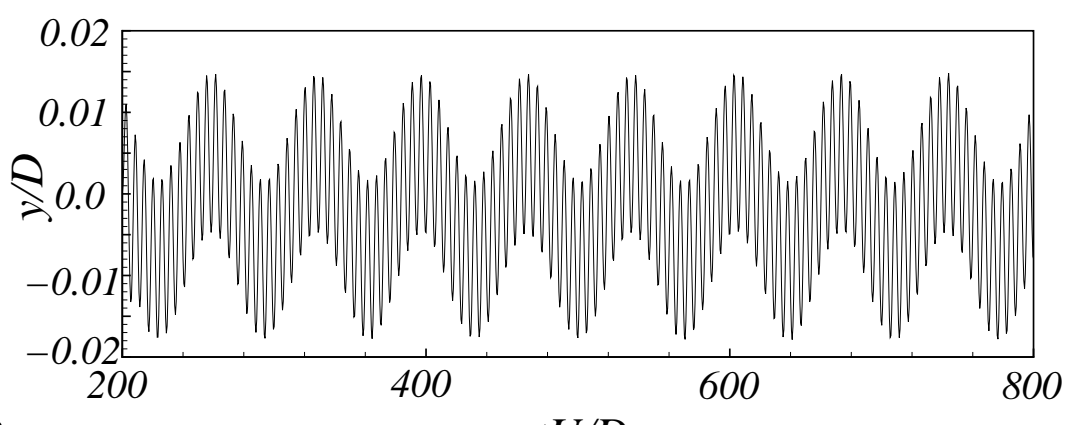

(c)

$t U / D$

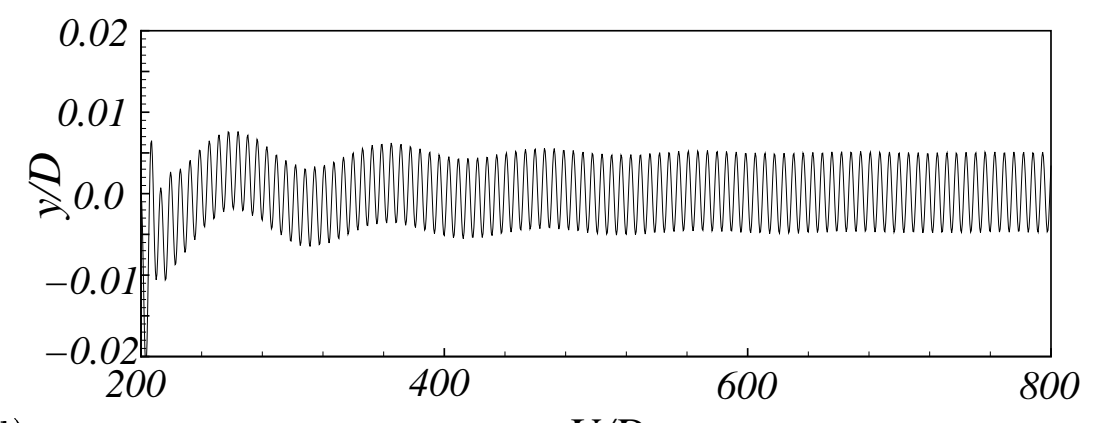

(d)

$t U / D$

Fig. 5. Transverse galloping responses: $\operatorname{Re}=250, \xi_{y}=0.0037, U_{y}=40, n_{y}=20$. (a) $\Lambda=1$. (b) $\Lambda=1.5$. (c) $\Lambda=2$. (d) $\Lambda=3$. 


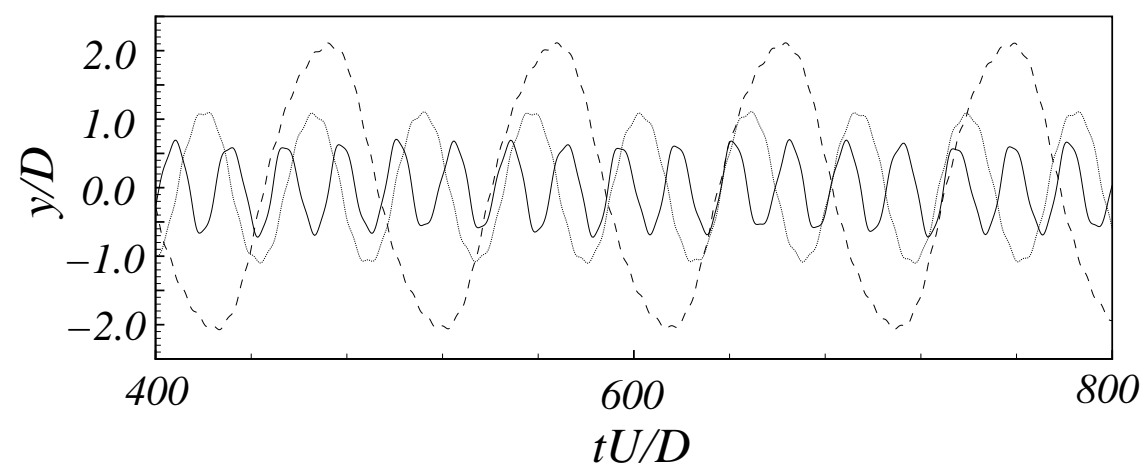

Fig. 6. Transverse galloping responses: $\operatorname{Re}=250, \xi_{y}=0.0037, \Lambda=1, n_{y}=10$. (a) $U_{y}=20$. (b) ......... $U_{y}=40$. (c) $---U_{y}=80$.

To investigate the effect of increasing reduced velocity on galloping, the motion of a section with $\Lambda=1$ was calculated at three values of reduced velocity. The time histories of the amplitude are shown in Figure 6. The results indicate an increase of amplitude with a corresponding increase in reduced velocity. This is consistent with the quasi-steady linear theory, equation (27), which predicts a decrease in the aerodynamic damping for an increase in the reduced velocity. Though it must be remembered that equation (27) does not allow us to predict the amplitude of the galloping oscillations, it can be inferred that, for a given body, a reduction in the damping may lead to an increase of the amplitude of the oscillations.

The quasi-steady theory predictions are only valid for small oscillations about the origin, as the amplitude of the oscillations increases non-linear flow behaviour not accounted for in the quasi-steady analysis may lead to the saturation of the large amplitude oscillations. Therefore whilst the quasi-steady theory predicts that the oscillations will become unbounded in time, it can be seen from Figure 6 that the oscillations are still bounded due to non-linear flow behaviour up to at least a reduced velocity of $U_{y}=80$.

A comparison between galloping and vortex excited resonance was also performed for the rectangular section of thickness ratio equal to 1.5. The Strouhal number for this section was estimated to be 0.158 . Therefore we considered a reduced velocity of $1 / 0.158 \approx 6$, which produced vortex lock-in oscillations as can be seen in Figure 7. For this resonant case, the oscillation amplitude is much smaller than those recorded for galloping at higher reduced velocities. A comparison of the flow patterns for vortex-induced resonance, galloping and a fixed structure is illustrated in Figure 8, where it is apparent that the longitudinal spacing between vortices is reduced for the vortex lock-in case when compared to the galloping and fixed structure cases. This illustrates the quasi-steady nature of the flow for the galloping structure since the vortex pattern and spacing are very similar to that of the fixed body, whereas the unsteady, high frequency vortex-induced motion exhibits a decrease in the separation of the shed vortices. 


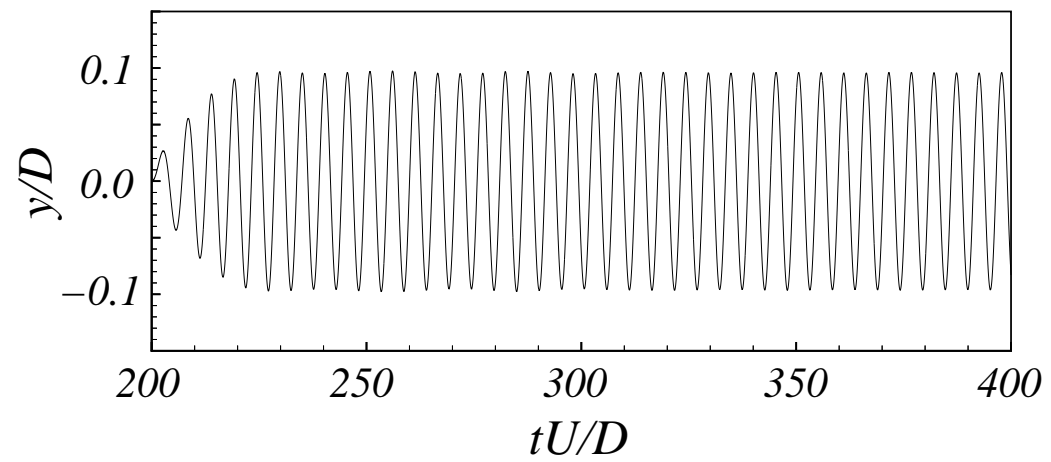

Fig. 7. Vortex-induced oscillation: $\operatorname{Re}=250, \xi_{y}=0.0037, U_{y}=6.0, n_{y}=10, \Lambda=1.5$.

The numerical results generated here are in agreement with the theory proposed by Parkinson (1971) to explain the transverse (or heave) galloping mechanism. He theorised that the motion of a rectangular structure led to the separated shear layers above and below the section becoming asymmetric. The closely lying shear layer produces a greater suction on the body than a more distant layer and therefore a pressure difference is formed across the body. Figure 9 shows the time-averaged flow field and pressure distribution, in terms of $C_{p}$, around the square section for increasing fixed angles of attack, $\alpha$. The shear layers are asymmetric when $\alpha=3^{\circ}$, causing a pressure difference which is not immediately apparent from $9(\mathrm{~b})$, but is implied by the variation of $C_{y}$ with $\alpha$ shown in Figure 4(a). The pressure applied on the bottom edge of the section is of a higher magnitude than that applied on the top edge. An increase to $6^{\circ}$ leads to a larger pressure difference due to the greater asymmetry of the shear layers. Since $\alpha=-\dot{y} / U$, the motion of the body leads to an asymmetry of the shear layers and a force in the direction of the motion of the body, thereby causing large amplitude, unstable oscillations. 


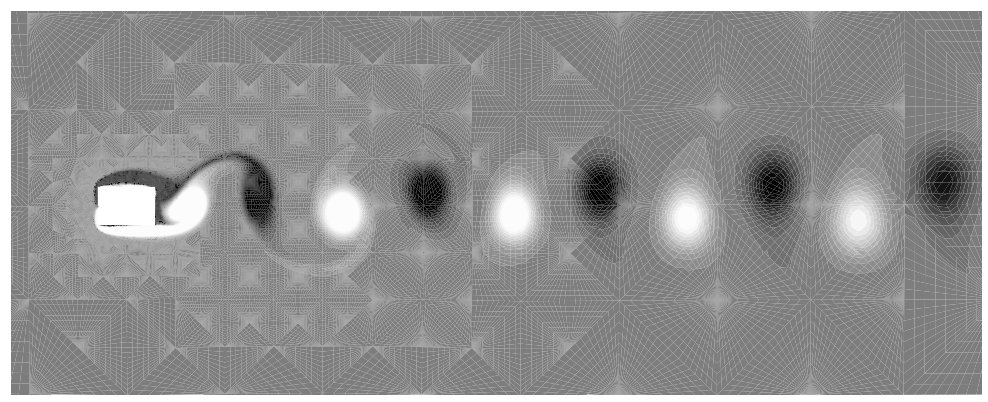

(a)

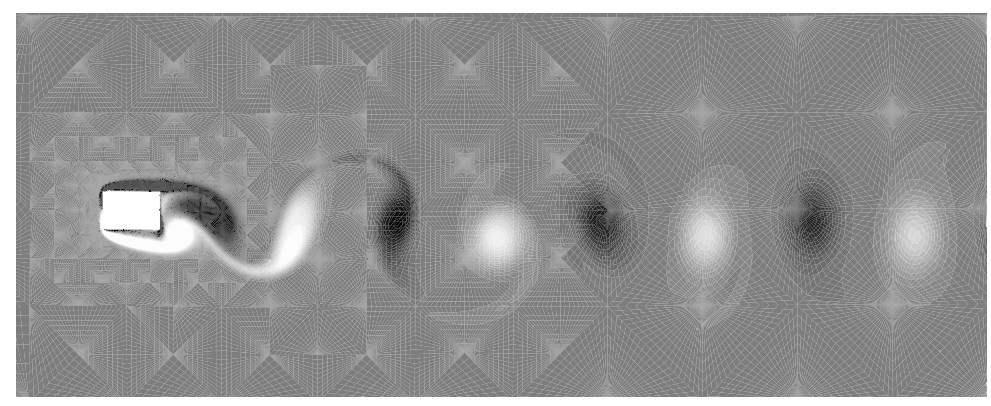

(b)

(c)

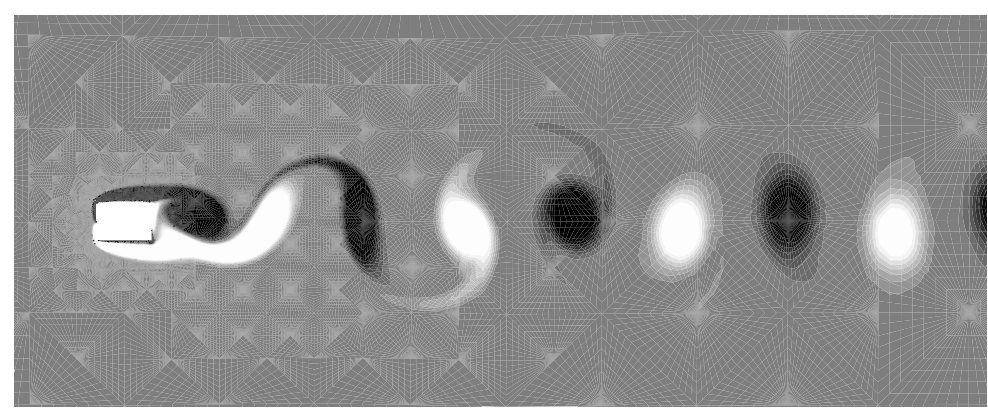

Fig. 8. Comparison of flow patterns for vortex-induced response, transverse galloping and fixed rectangular sections: $\operatorname{Re}=250, \xi_{y}=0.0037, \Lambda=1.5, n_{y}=10$. (a) Vortex-induced resonance, $U_{y}=6$ (b) Transverse galloping, $U_{y}=40$, (c) Fixed. 

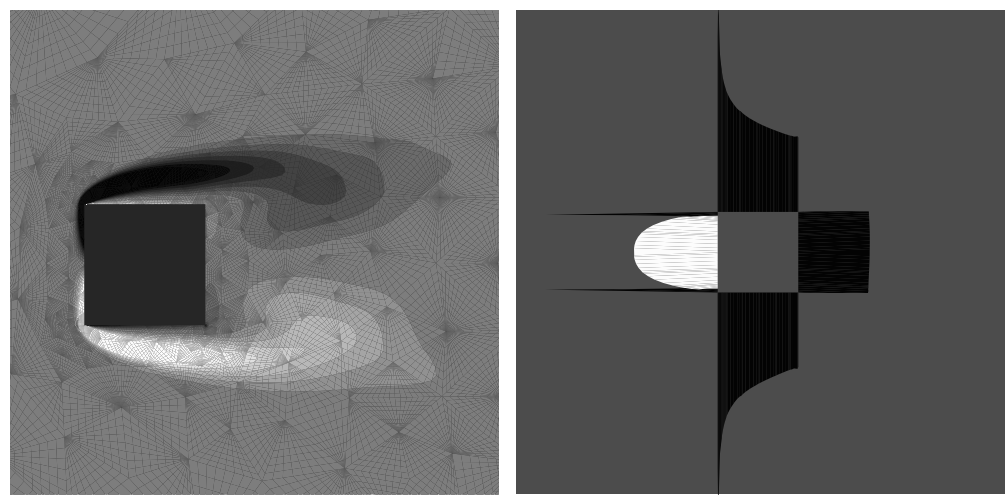

(a) $\alpha=0^{\circ}$
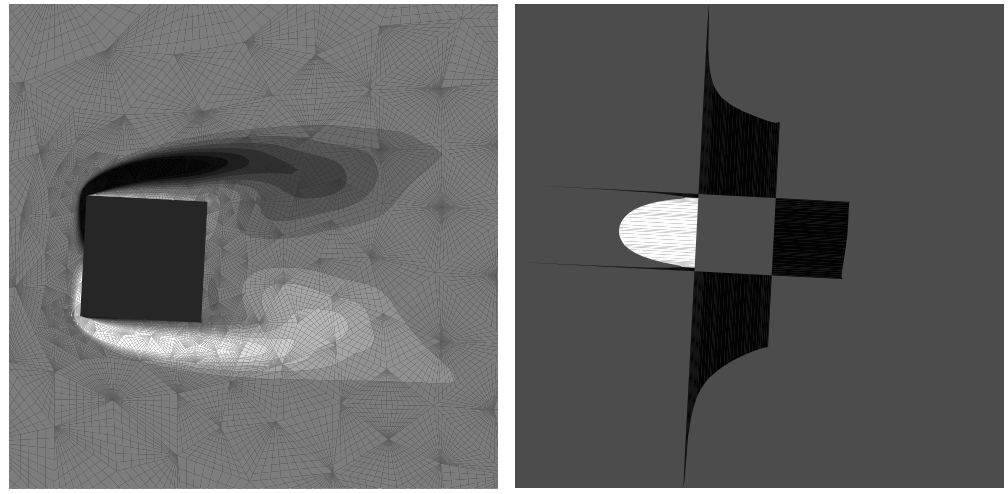

(b) $\alpha=3^{o}$
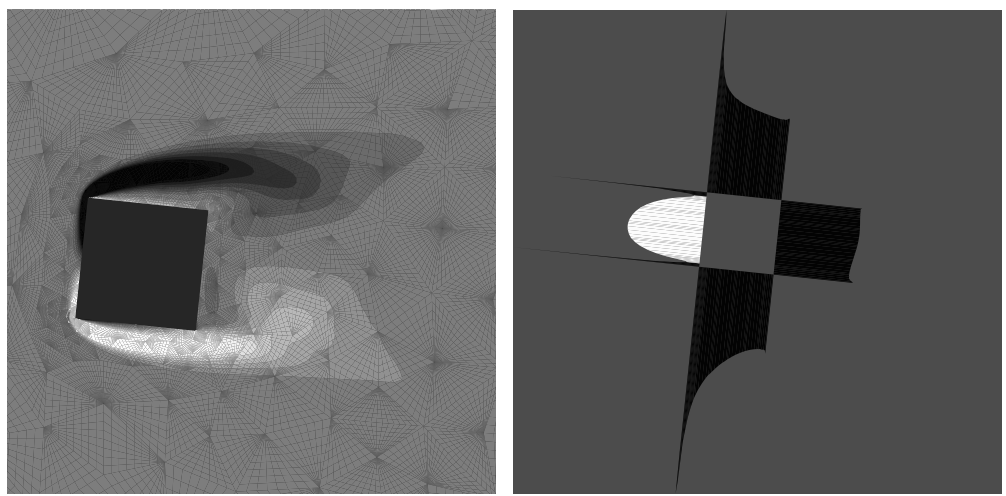

(c) $\alpha=6^{\circ}$

Fig. 9. Averaged vorticity field, pressure distribution (white indicating positive pressure and black negative) for a fixed square section at $R e=250$ 
For higher aspect ratio rectangular sections, $\Lambda<2.5$, the top shear layer reattaches or nears reattachment for positive displacements about $\alpha=0$. This results in a pressure difference which exerts a force in the opposite direction to the motion of the section. The aerodynamic damping is therefore positive and the oscillations are stable. The re-attaching shear layers and pressure differential are illustrated in Figure 10 for a rectangular section of $\Lambda=4$ and $\mathrm{Re}=250$ at various effective angles of attack $\alpha$.
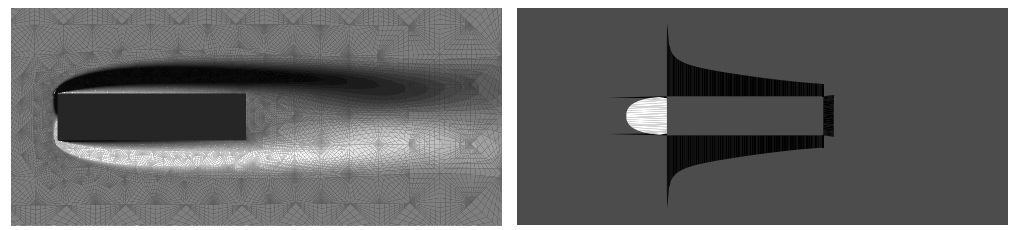

(a) $\alpha=0^{\circ}$
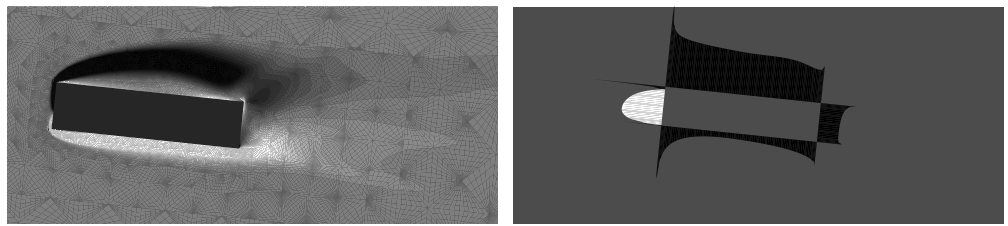

(b) $\alpha=6^{o}$
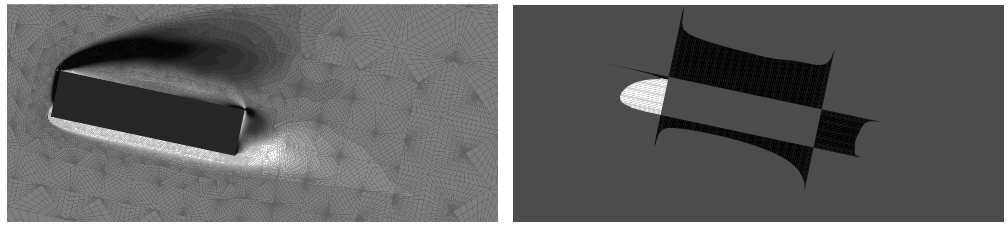

(c) $\alpha=12^{\circ}$

Fig. 10. Averaged vorticity field, pressure distribution (white indicating positive pressure and black negative) for a fixed rectangular section of $\Lambda=4$ at $\operatorname{Re}=250$

Figure 11 shows the vorticity field around a moving rectangular section of aspect ratio $\Lambda=1$ undergoing a vertical galloping motion, and the resulting pressure distribution in terms of $C_{p}$. The displacement time history of the section is also shown in Figure 11, with the position of the body at the various snapshots highlighted. It can be seen that at the points (b) and (d) the body is close to its maximum displacement and therefore $\dot{y}$ and $\alpha$ are approximately zero. Consequently the shear layers are predominantly symmetric, resulting in a minimal force acting on the body. In contrast to this the asymmetric vorticity fields in Figures 11(a) and 11(c), where $\alpha$ is negative and positive respectively, indicate the possibility of a pressure difference acting in the direction of motion. Therefore, the structure is taking energy from the fluid, resulting in unstable, growing oscillations. 


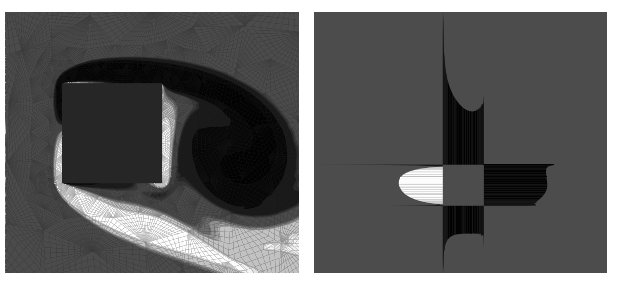

(a)
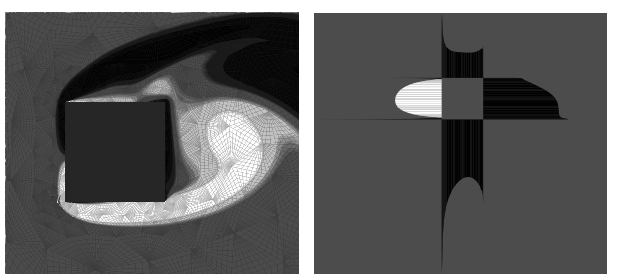

(c)

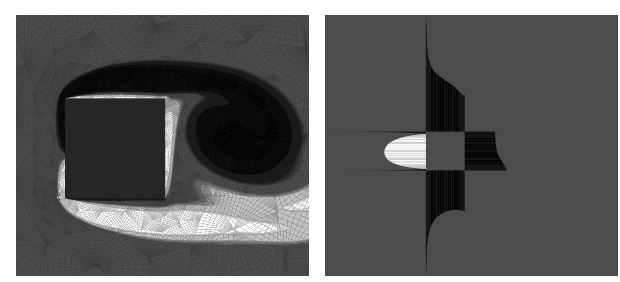

(b)
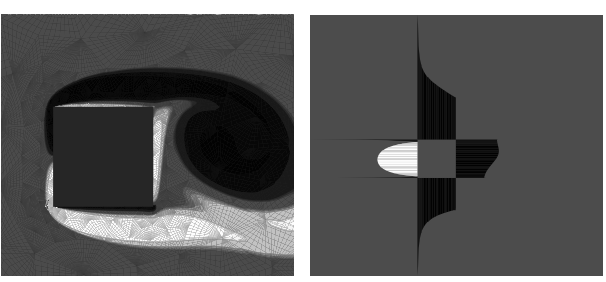

(d)

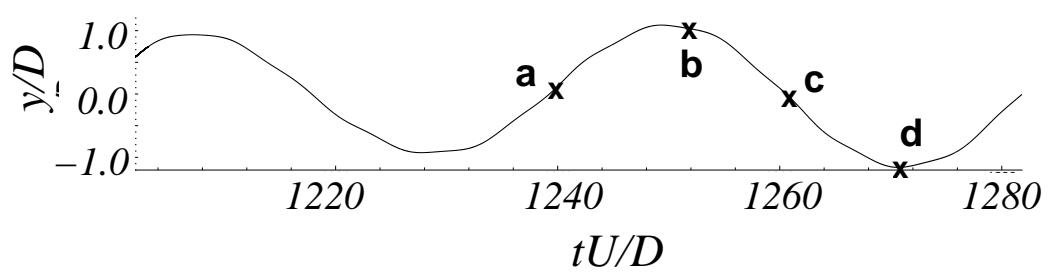

(e) Transverse displacement

Fig. 11. Vorticity field, pressure distribution (white indicating positive pressure and black negative) and displacement time-history of galloping rectangular section: $\Lambda=1, \operatorname{Re}=250$, $\xi_{y}=0.0037, n_{y}=10, U_{y}=40$ 
(a)

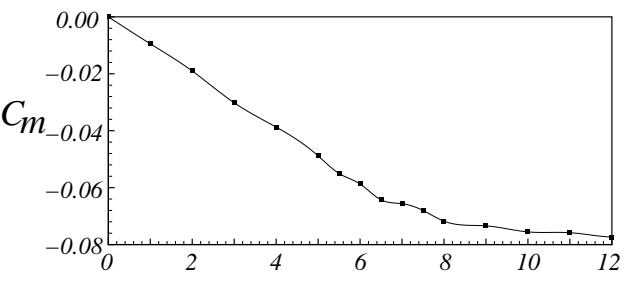

(c)

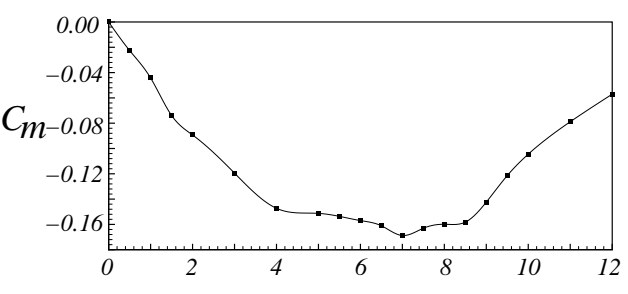

(e)

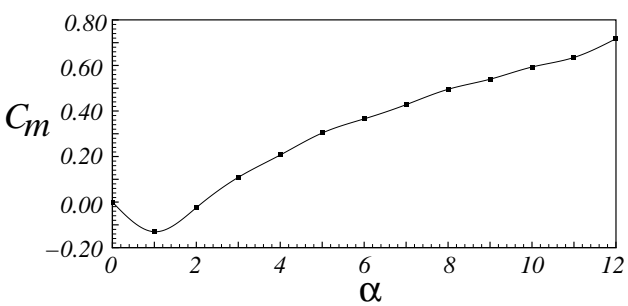

(b)

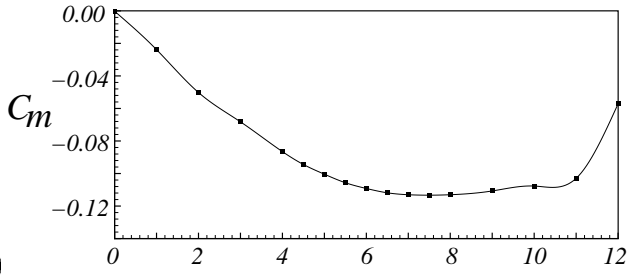

(d)

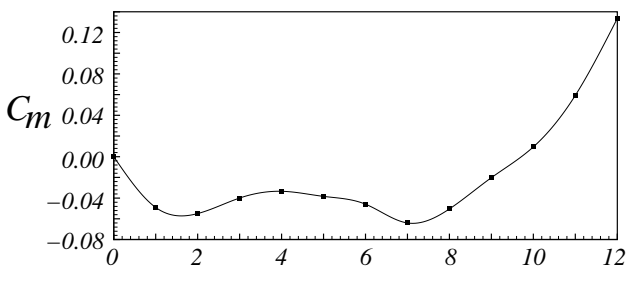

(f)

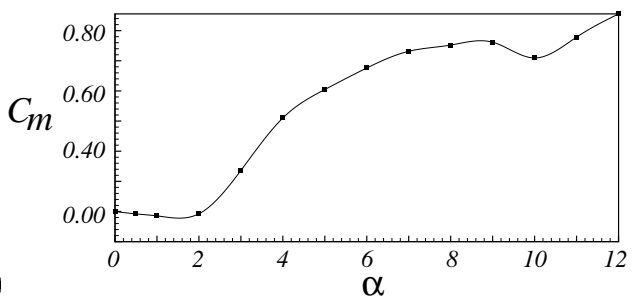

Fig. 12. Moment coefficient, $C_{M}$, against angle of attack $\alpha$ (degrees). (a) $\Lambda=1$. (b) $\Lambda=1.5$. (c) $\Lambda=2$. (d) $\Lambda=3$. (e) $\Lambda=4$. (f) $\Lambda=5$.

\section{Numerical Investigation of Rotational Galloping}

The previous numerical experiments undertaken in Section 5 to evaluate $\partial C_{y} / \partial \alpha$ also provide the variations of $C_{M}$ against $\alpha$. These results are shown in Figure 12 and will be compared with the dynamic numerical simulations discussed below. The values of $d C_{M} /\left.d \alpha\right|_{\alpha=0}$ are also given in Table 2. The data in Figure 12 and Table 2 indicates that all the sections considered have the potential to gallop as the value of $d C_{M} / d \alpha$ about $\alpha=0$ is negative for all cases. This is consistent with the findings of other authors (Blevins, 1990; Nakamura \& Mizota, 1975; Luo et al., 1998), who consequently went on to show experimentally that bodies with $d C_{M} / d \alpha<0$ are dynamically unstable.

The dynamic numerical experiments were performed by allowing the section to rotate when acted upon by an oncoming free-stream parallel with the $x$-axis. For the square section, $\Lambda=1$, we see from Figure 13 that there are two distinct frequencies appearing in the time history of the non-dimensional oscillation amplitude, the high frequency relates to the oscillating force produced by the vortex shedding, and the low frequency indicates a galloping motion. The low amplitude of the galloping oscillations is expected from the very small value of $d C_{M} / d \alpha$ for this geometry.

For all sections of aspect ratio greater than one, the numerical results in Figures 14 to 17 show large amplitude galloping oscillations for a reduced velocity of 
Table 2

\begin{tabular}{|c||c|c|c|c|c|c|}
\hline$\Lambda$ & 1 & 1.5 & 2 & 3 & 4 & 5 \\
\hline$\left.\frac{d C_{M}}{d \alpha}\right|_{\alpha=0}$ & -0.516 & -1.49 & -2.46 & -3.21 & -9.74 & -2.06 \\
\hline
\end{tabular}

Variation of the moment coefficient against angle of attack in radians about a zero effective angle of attack for rectangular sections of increasing aspect ratio.

40. For the cases with no structural damping, $\xi_{\theta}=0$, the amplitude appears to be unbounded. However, this cannot be confirmed due to the limitations of the computational code, which is not valid for very high amplitudes of displacement due to the implementation of the boundary conditions ( $\mathrm{Li}$ et al., 2002). The galloping oscillations have been limited by increasing the damping ratio, $\xi_{\theta}$, to a sufficiently large value, as seen in sub-plots (b) of Figures 14 to 17.

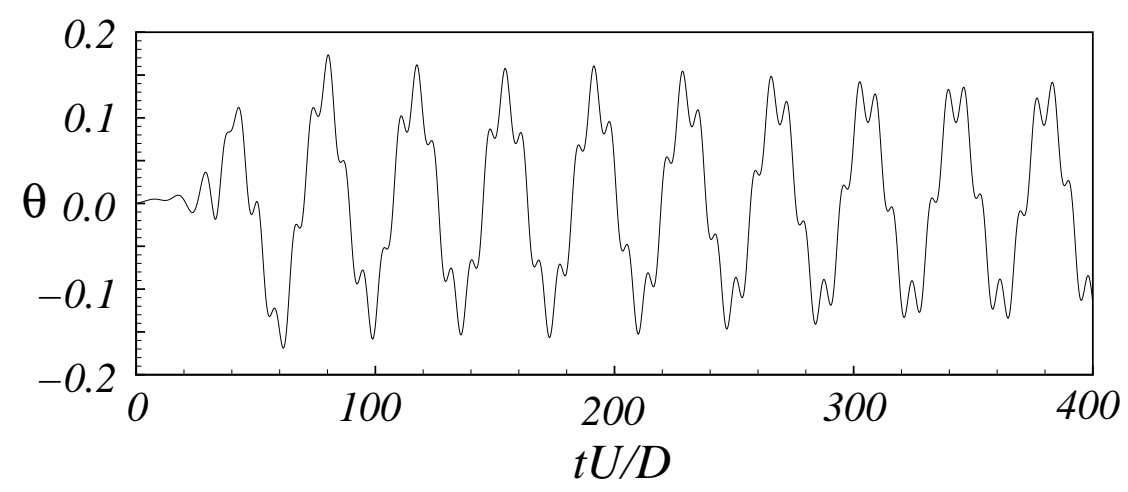

Fig. 13. Free rotation: $\Lambda=1, \xi_{\theta}=0, U_{\theta}=40, n_{\theta}=100$. 


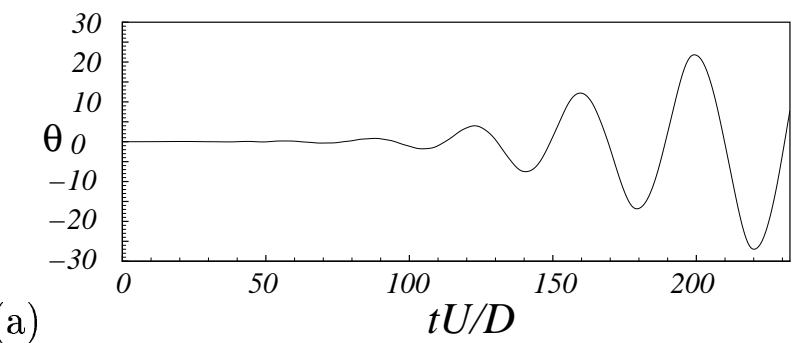

(a)

$t U / D$

(b)

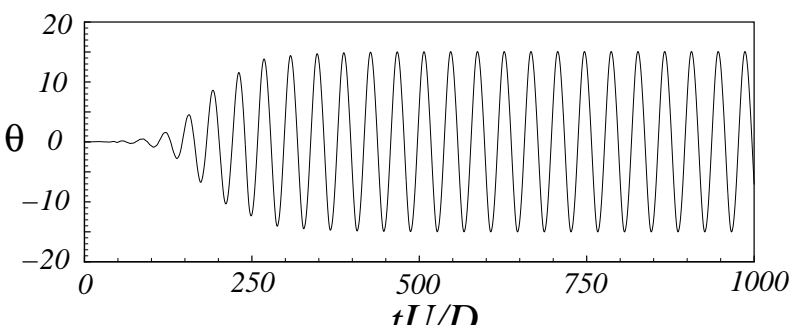

Fig. 14. Free rotation: $\operatorname{Re}=250, \Lambda=2, U_{\theta}=40, n_{\theta}=100$; (a) $\xi_{\theta}=0$, (b) $\xi_{\theta}=0.1$.

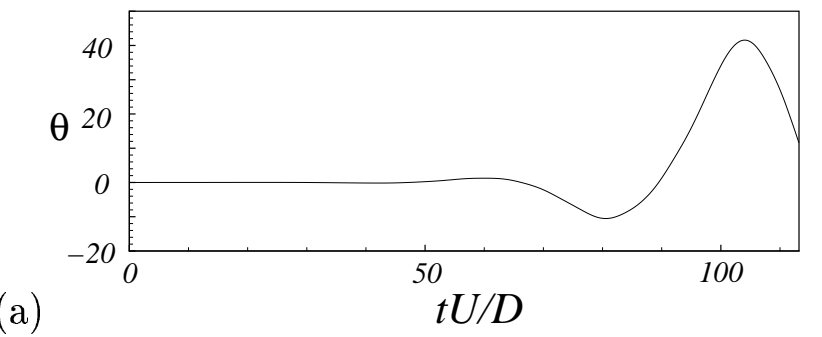

(a)

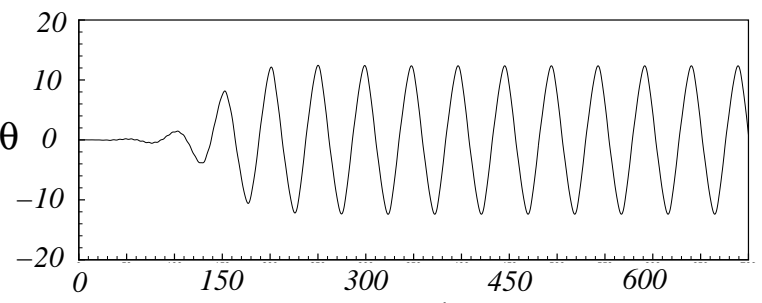

(b)

$t U / D$

Fig. 15. Free rotation: $\operatorname{Re}=250, \Lambda=3, U_{\theta}=40, n_{\theta}=100$; (a) $\xi_{\theta}=0$, (b) $\xi_{\theta}=0.5$. 

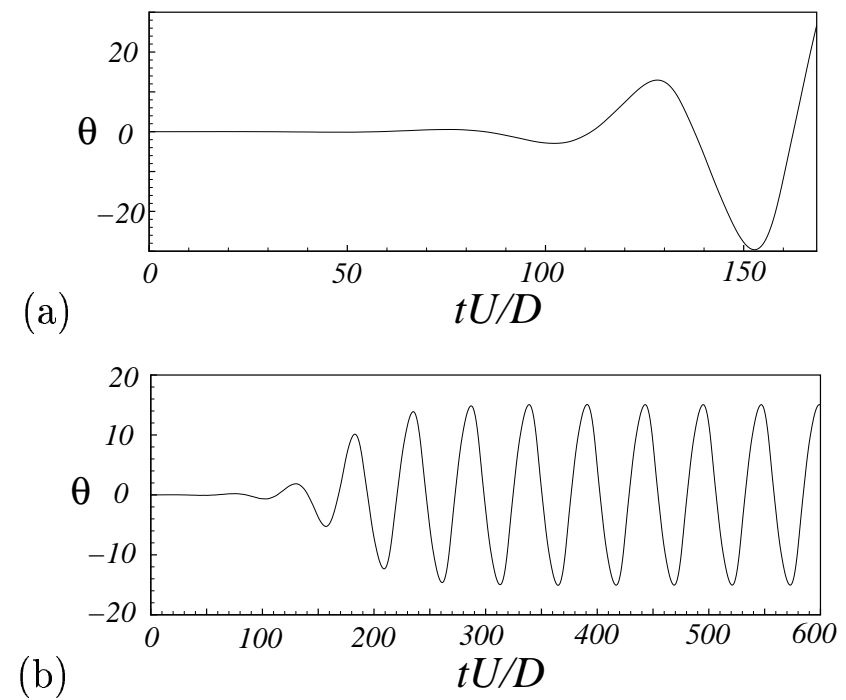

Fig. 16. Free rotation: $\operatorname{Re}=250, \Lambda=4 U_{\theta}=40, n_{\theta}=400$, (a) $\xi_{\theta}=0$, (b) $\xi_{\theta}=0.25$.

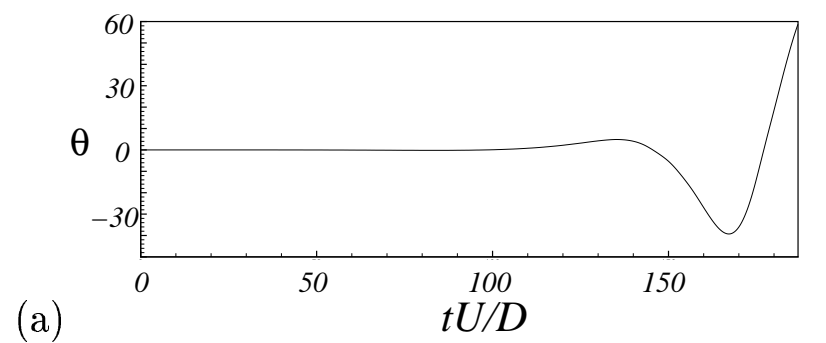

(a)

$$
\begin{gathered}
100 \\
t U / D
\end{gathered}
$$

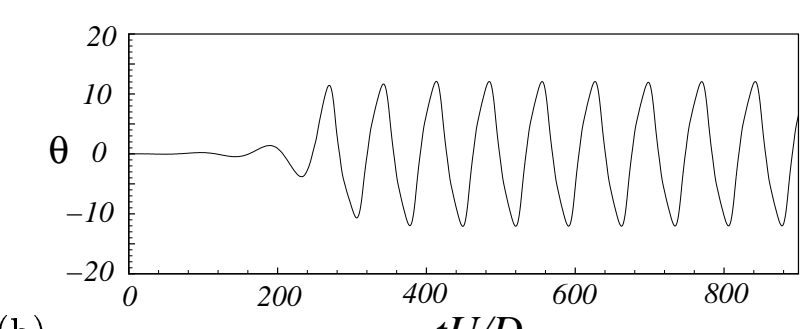

(b)

$t U / D$

Fig. 17. Free rotation: $\operatorname{Re}=250, \Lambda=5, U_{\theta}=40, n_{\theta}=400$, (a) $\xi_{\theta}=0$, (b) $\xi_{\theta}=0.6$. 
The motion of a rectangular section with $\Lambda=4, n_{\theta}=400, \xi_{\theta}=0.25, U_{\theta}=40$ and $R e=250$ can be seen in Figure 18, where the flow field is represented as vorticity contours and the pressure distribution given in terms of $C_{p}$. The large pressure variations at time levels (a) and (c) occur when the rotational displacement is at its greatest and together with the time history of the displacement in Figure 18(e) it can be seen that the moment and displacement are largely in phase. However, it is the component of the moment which is in phase with the velocity that causes the instability of the motion. The vorticity fields associated with the component of the moment in the direction of motion can be seen at time levels (b) and (d), where it is apparent that the pressure distribution is such that the moment of the section is in the direction of motion of the body, thus causing unstable oscillations.
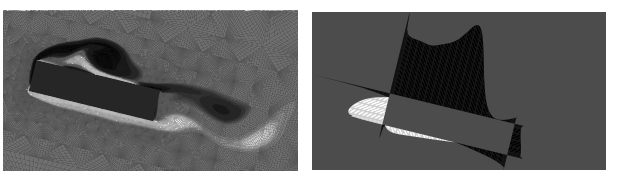

(a)
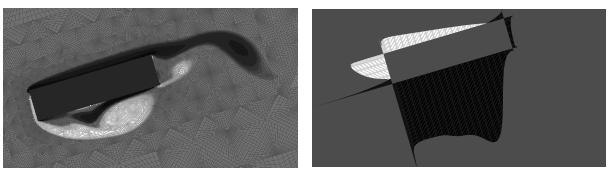

(c)

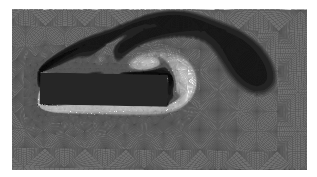

(b)

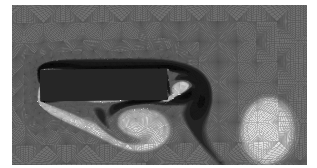

(d)
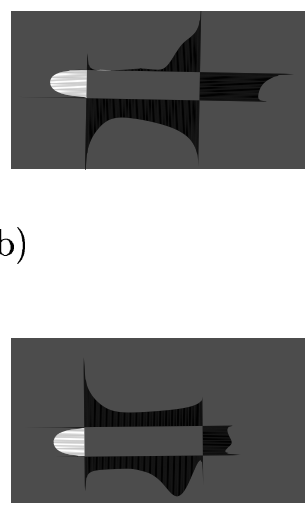

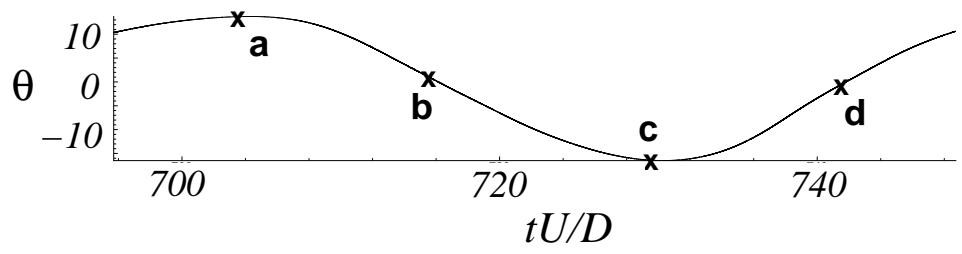

(e) Rotational displacement

Fig. 18. Vorticity field, pressure distribution (white indicating positive pressure and black negative) and displacement time-history of galloping rectangular section: $\Lambda=4, \operatorname{Re}=250$, $\xi_{\theta}=0.25, n_{\theta}=400, U_{\theta}=40$ 


\section{Conclusions}

We have reviewed the quasi-steady analysis of transverse and rotational galloping and presented numerical simulations which dynamically predict the galloping modes. The numerical results for both transverse and rotational galloping are consistent with the predictions of quasi-steady analysis although only a qualitative agreement is observed.

Much of the work is in qualitative agreement with experimental data (Parkinson, 1971; Blevins, 1990; Washizu \& Ohya, 1978) obtained at a higher Reynolds numbers $\left(\approx 1 \times 10^{5}\right)$ than the current numerical data $(=250)$. This observation may well be related to the Reynolds number independent separation point of the rectangular structures considered although we note that the reattachment length of a separated shear layer is Reynolds number dependent (Parkinson, 1971).

To conclude, a detailed analysis of low Reynolds number flow past different aspect ratio rectangular cylinders which are allowed to either translate vertically or rotate has been performed.

\section{Acknowledgements}

Thanks are due to EPSRC for their direct financial support of this research through research grant GR/L48096 and to BMT Fluid Mechanics Ltd for the supply of experimental data and Flint \& Neil Partnership for helpful discussions.

\section{Appendix A: Spatial and Temporal Convergence}

Spatial and temporal convergence is illustrated for the case of a rectangular body with an aspect ratio $\Lambda=4$ undergoing large amplitude rotational galloping motion. The flow and structure are described by the following parameters: $\Lambda=4$, $n_{\theta}=400, \xi_{\theta}=0.25, U_{\theta}=40$ and $\operatorname{Re}=250$. Time histories of the rotational displacement for increasing computational time increments are shown in Figure 19. The three time histories are indistinguishable, with the two smallest time increments differing by approximately $0.2 \%$. The percentage error between the results can be seen in Table 3.

Time histories of the rotational displacement for increasing polynomial order of the expansion basis are shown in Figure 20. Spatial convergence is illustrated by the time histories when using polynomial orders of 4 and 6 . The histories differ by 


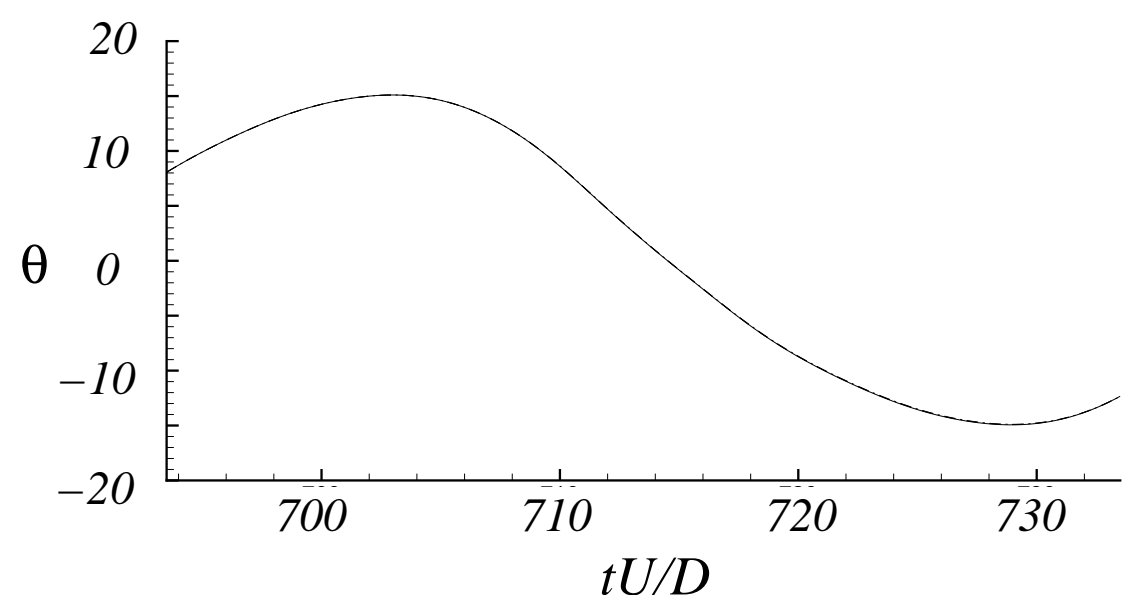

Fig. 19. Time history of rotational displacement for: $-\Delta t=0.0005,---\Delta t=0.001$ and $-\cdot-\cdot-\Delta t=0.002$

approximately $0.1 \%$. The percentage error between the results can be seen in Table 4.

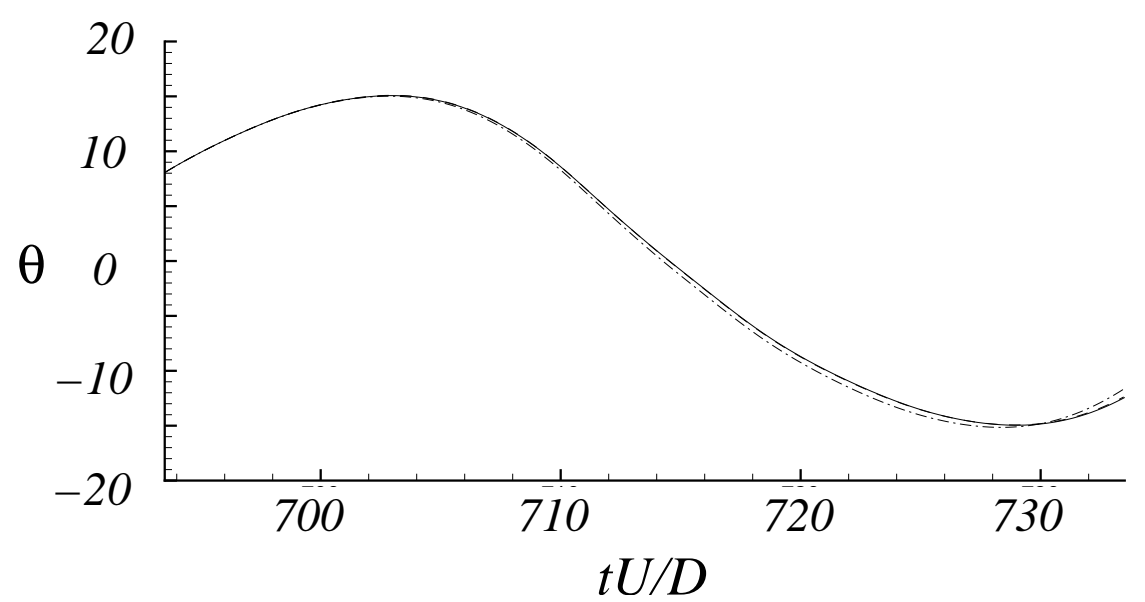

Fig. 20. Time history of rotational displacement for: $-p=6,---p=4$ and $-\cdot-\cdot-$ $p=2$

Table 3

\begin{tabular}{|c|c|c|}
\hline$\Delta t$ & $\theta$ & $\%$ difference to $\Delta t=0.0005$ \\
\hline 0.002 & 13.4933 & 0.42 \\
\hline 0.001 & 13.5209 & 0.21 \\
\hline 0.0005 & 13.5497 & 0 \\
\hline
\end{tabular}

Temporal convergence of the displacement of a rotating body with an aspect ratio of $\Lambda=4$ at $t U / D=725$. 
Table 4

\begin{tabular}{|c|c|c|}
\hline Polynomial Order $(\mathrm{P})$ & $\theta$ & \% difference to $P=6$ \\
\hline 2 & 14.0421 & 3.6 \\
\hline 4 & 13.5497 & 0.12 \\
\hline 6 & 13.5331 & 0 \\
\hline
\end{tabular}

Spectral element spatial convergence of the displacement of a rotating body with an aspect ratio of $\Lambda=4$ at $t U / D=725$. 


\section{References}

Anagnostopoulos, P. 1994 Numerical investigation of response and wake characteristics of a vortex-excited cylinder in a uniform stream. Journal of Fluids and Structures 8, 367-390.

Bearman, P.W. 1984 Experiments on flow-induced vibrations of a square-section cylinder. In: Paidoussis, M. P. (Ed.), Symposium on Flow-induced Vibrations. Vol. 1.

Blevins, R.D. 1990 Flow-induced Vibration. Van Nostrand Reinhold, New York. den Hartog, J.P. 1956 Mechanical Vibrations, 4th Edition. McGraw-Hill, New York.

Deniz, S. 1997 Oscillating rectangular and octagonal profiles: interaction of leadingand trailing-edge vortex formation. Journal of Fluids and Structures 11, 3-31.

Kozakiewicz, A., Sumer, B.M. \& Fredsoe, J. 1992 Spanwise correlation on a vibrating cylinder near a wall in oscillatory flows. Journal of Fluids and Structures 6, 371392.

Li, L., Sherwin, S.J. \& Bearman, P.W. 2002 A moving frame of reference algorithm for fluid/structure interaction of rotating and translating bodies. International Journal of Numerical Methods in Fluids 38, 207-223.

Luo, S.C., Chew, Y.T., Lee, T.S. \& Yazdani, M.G. 1998 Stability to translational galloping vibration of cylinders at different mean angles of attack. Journal of Sound and Vibration 215, 1183-1194.

Nakamura, Y. \& Mizota, T. 1975 Torsional flutter of rectangular prisms. J. Eng. Mech. Div., Am. Soc. Civil Engrs. EM 2 (101), 125-142.

Nakamura, Y., Nakashima, M. 1986 Vortex excitation of prisms with elongated rectangular, $h$ and $\vdash$ cross-sections. Journal of Fluid Mechanics 163, 149-169.

Nakamura, Y. \& Tomonari, Y. 1977 Galloping of rectangular prisms in a smooth and a turbulent flow. Journal of Sound and Vibration 52, 233-241.

Naudascher, E. \& Rochwell, D. 1994 Flow-induced Vibration-An engineering guide. A. A. Balkema, Rotterdam.

Nomura, T. 1993 Finite element analysis of vortex-induced vibrations of bluff cylinders. Journal of Wind Engineering and Industrial Aerodynamics 46, 587-594.

Nomura, T. \& Hughes, T.J.R. 1992 An arbitrary lagrangian-eulerian finite element method for interaction of fluid and a rigid body. Computer Methods in Applied Mechanics and Engineering 95, 115-138.

Parkinson, G. 1971 Wind-induced instability of structures. Phil. Trans. Roy. Soc. Lond. A. 269, 395-409.

Piperno, S. 1998 Numerical simulation of aeroelastic instabilities of elementary bridge decks. INRIA, Rapport de recherche, 3549th Edition.

Robertson, I., Sherwin, S.J. \& Bearman, P.W. 2001 Prediction of flutter instability due to cross winds of the second forth road bridge. In: ECCOMAS 2001.

Robertson, I., Sherwin, S.J. \& Bearman, P.W. 2002 Flutter instability prediction techniques for bridge deck sections. Under review for publication in International Journal for Numerical Methods in Fluids .

Scanlan, R. 1979 On the state of stability considerations for suspended-span bridges under wind. In: Proceedings IUTAM-IAHR Symposium, Karlsruhe, Germany. pp. 
$595-618$.

Scanlan, R. \& Tomko, J. 1971 Airfoil and bridge deck flutter derivatives. Journal of the Engineering Mechanics Division, ASCE EM6 (97), 1717-1737.

Schulz, K. \& Kallinderis, Y. 1998 Unsteady flow structure interaction for incompressible flows using deformable hybrid grids. Journal of Computational Physics 143, 569-597.

Sisto, F. 1953 Stall-flutter in cascades. Journal of Aeronautical Science 20, 598-604.

Steinman, D.B. \& Watson, S.R. 1957 Bridges and their Builders. Dover, New York. Sumer, B.M., Fredsoe, J. \& Jensen, K. 1994 A note on spanwise correlations on a freely vibrating cylinder in oscillatory flow. Journal Fluids and Structures 8, 231-238.

Washizu, K. \& Ohya, A. 1978 Aeroelastic instability of rectangular cylinders in a heaving mode. Journal of Sound and Vibration 59, 195-210.

Washizu, K., Ohya, A., Otsuki, Y. \& Fujii, K. 1980 Aeroelastic instability of rectangular cylinders in a torsional mode due to a transverse wind. Journal of Sound and Vibration 72, 507-521.

Wei, R., Sekine, A. \& Shimura, M. 1995 Numerical analysis of 2D vortex-induced oscillations of a circular cylinder. International Journal for Numerical Methods in Fluids 21, 993-1005. 